\title{
Bilgepumpe, Zuckerrohrmühle, Hebewerk: Technische Geräte und Maschinen in der neulateinischen Jesuiten-Lehrdichtung
}

In seiner um das Jahr 335 vor Christus entstandenen Poetik formuliert Aristoteles eine der bekanntesten und folgenreichsten Aussagen zum hexametrischen Lehrgedicht. ${ }^{1}$ Homer und Empedokles, so schreibt er, hätten nichts gemeinsam außer dem Versmaß: deshalb sei letzterer eher Naturforscher denn Dichter. ${ }^{2}$ Die Frage, ob Lehrdichtung überhaupt als Dichtung anzusprechen sei, ist seit Aristoteles' Verdikt immer wieder Gegenstand von Diskussionen gewesen. Zentrale Punkte in der Kontroverse um das Lehrgedicht als „Problem der Poetik“3 ${ }^{\text {*3 }}$ waren dabei neben seinem ,amimetischen' Charakter, dem man durch eine Weitung des Mimesis-Begriffs zu entgehen suchte, ${ }^{4}$ auch das Mit- und Gegeneinander von sprödem fachwissenschaftlichem Inhalt und hochpoetischer Form, ${ }^{5}$ das für die Lehrdichtung der Antike nicht selten als ,Spannungsverhältnis‘ wahrgenommen und beschrieben wurde. ${ }^{6}$ Spätestens mit dem Aufkommen einer wissenschaftlichen Spezialliteratur

1 Für die Durchsicht des Aufsatzes und wertvolle Anregungen danke ich Dr. Jochen Walter (Mainz).

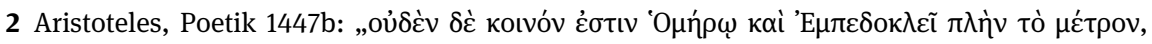

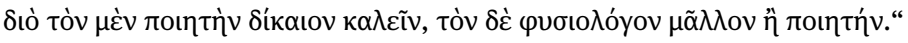

3 Bernhard Fabian: Das Lehrgedicht als Problem der antiken und modernen Poetik. In: Die nicht mehr schönen Künste. Hg. von Hans R. Jauss. München 1968 (Poetik und Hermeneutik 3), S. 67-89; Robert M. Schuler, John G. Fitch: Theory and context of the didactic poem: Some classical, mediaeval, and later continuities. In: Florilegium 5 (1983), S. 1-43, hier S. 21-29.

4 So etwa Girolamo Fracastoro in seinem Dialog Naugerius sive de poetica dialogus (in der Ausgabe der Opera omnia, Venedig 1555; moderne Ausgabe: Hg. von Ruth Kelso, Murray Wright Bundy. University of Illinois 1924), S. 156 D. Dazu Fabian (Anm. 3), S. 83. Weitere frühneuzeitliche Kritiker, die das Mimesis-Prinzip nicht allein auf Menschen beschränken, sind Bernardino Tomitano und Sperone Speroni) (vgl. dazu Baxter Hathaway: The age of Criticism. The Late Renaissance in Italy. Ithaca, NY 1962, S. 67-69) und Christopher Stay: De poesi didascalica dialogus. Rom 1792, S. xi-xii (vgl. dazu Claudia Schindler: Didactic Poetry as Elitist Poetry: Christopher Stay's De poesia didascalica dialogus in the Context of Classical and Neo-Latin Didactic Discourse. In: Neo-Latin and the Vernaculars. Bilingual interactions in the Early Modern Period. Hg. von Florian Schaffenrath, Alexander Winkler. Leiden, Boston 2019, S. 232-250, hier S. 237-239).

5 Katharina Volk: The poetics of Latin didactic. Lucretius, Vergil, Ovid, Manilius. Oxford 2002, S. 55, besonders deutlich formuliert Manil. 1,20-23: „bina mihi positis lucent altaria flammis / ad duo templa precor duplici circumdatus aestu / carminis et rerum.“

6 Vgl. Egert Pöhlmann: Charakteristika des römischen Lehrgedichts. In: ANRW I,3 (1973), S. 813-901, hier S. 834-835. 
in Prosa wurde die Frage nach dem Verhältnis von ,Dichtung‘ und ,Lehre` virulent - insbesondere die Frage, mit welcher Intention ein Autor sich dafür entschieden haben könnte, besonders unzugängliche Inhalte der Fachwissenschaft einer poetischen Gestaltung zu unterziehen.

Beschreibungen von Maschinen und technischen Gerätschaften stellen im Kontext der poetischen Gestaltung fachwissenschaftlicher Gegenstände eine besondere Herausforderung dar. In der antiken Lehrdichtung begegnet Derartiges nur sehr vereinzelt. Hesiod beschreibt in den Erga kai hemerai den Bau eines Pfluges. ${ }^{7}$ Vergil greift diese Beschreibung im ersten Buch der Georgica mit einer beinahe wörtlichen Übersetzung auf. 8 Als Beschreibung eines Artefakts im allerweitesten Sinne kann man ferner die Schilderung der Vorrichtung ansehen, in die der für das Mirakel der Bugonie präparierte Rindskadaver hineingelegt wird. ${ }^{9}$

Während der Befund für das antike Lehrgedicht eher spärlich ausfällt, steigt die Frequenz der Beschreibungen von Geräten und Maschinen insbesondere im neulateinischen Jesuiten-Lehrgedicht des späteren siebzehnten und achtzehnten Jahrhunderts so stark an, dass sie geradezu zu einem generischen Marker dieser Dichtungen werden. Die Darstellungen befassen sich dabei sowohl mit Objekten des häuslichen Gebrauchs, zum Beispiel den Gerätschaften und Gefäßen für die Kaffee- ${ }^{10}$ und Teezeremonie, ${ }^{11}$ als auch mit größeren Apparaturen wie der ,Produktionsstraße‘ zur Herstellung von Zucker ${ }^{12}$ oder Schokoladeneis, ${ }^{13}$ mit mechanischen Geräten wie einer Seil- und Zahnradwinde für den Stapellauf von Riesenschiffen, ${ }^{14}$ Hilfsmitteln für die naturwissenschaftliche Forschung, etwa Mikroskop ${ }^{15}$ und Vakuum-Kugeln, ${ }^{16}$ schließlich mit techni-

7 Hes. Erga 414-436.

8 Verg. Georg. 1,169-175.

9 Verg. Georg. 4,294-307.

10 Guglielmo Massieu: Caffaeum, carmen. In: Poemata didascalica. Hg. von François Oudin. Bd. 1. ${ }^{2}$ Paris 1813, S. 156.

11 Pierre Petit: Thia Sinensis, carmen (1685). In: Poemata didascalica. Hg. von François Oudin. Bd. 1. ${ }^{2}$ Paris 1813, S. 32.

12 Tommaso Strozzi: De mentis potu seu de cocolatis opificio. In: T. Strozzi, Poemata varia. Neapel 1689, S. 25-29.

13 Strozzi, De cocolatis opificio (Anm. 12), S. 53-56.

14 Nicolai Parthenii Giannettasii Neapolitani e Societate Jesu opera omnia poetica, Tomus Secundus complectens Piscatoria, Nautica et Halieutica. Neapel 1715, S. 84-85.

15 Melchior de Polignac: Antilucretius sive de deo et natura libri novem, opus posthumum. Paris 1747, S. 324.

16 Bernardo Zamagna: Navis aëria et elegiarum monobiblos. Rom 1768 (Neuedition: Bernardo Zamagna: Navis aëria. Hg. von Diane Bitzel. Frankfurt a.M. 1997), 1, 279-290. 
schen Spielereien wie der machina electrica ${ }^{17}$, der phiala Leidensis ${ }^{18}$ und dem cymbalum electricum. ${ }^{19}$ Generell lässt sich dabei feststellen, dass in den Lehrdichtungen des siebzehnten Jahrhunderts vor allem Maschinen und Gegenstände des allgemeinen Gebrauchs beschrieben werden, während Geräte für naturwissenschaftliche Forschung (Mikroskop und Vakuum-Kugeln) und technische Spielereien (cymbalum electricum) in den Lehrdichtungen des achtzehnten Jahrhunderts ihren Platz finden.

Wenngleich die Geräte-Beschreibungen der neulateinischen Lehrdichtung Gegenstände unterschiedlichster Größe, Form und Funktion betreffen, so ist ihnen doch eines gemeinsam. Es sind Artefakte, die aus verschiedenen Gründen ungewöhnlich sind: Sie sind besonders exotisch und rar, besonders groß, besonders funktional oder besonders komplex: und sie sind dem zeitgenössischen Rezipienten, dies ist zumindest die Vorannahme des Lehrdichters, nicht bekannt. Die Frage nach dem Verhältnis von ,Lehre‘ und ,Dichtung‘ hängt hier somit mit der Frage nach der ,Diskursivierung von Neuem‘ eng zusammen. ${ }^{20}$ Daraus ergeben sich, auf unterschiedlichen Ebenen, zwei grundsätzliche Problem- beziehungsweise Fragestellungen. Sie sollen im Folgenden kurz skizziert werden, bevor ich anhand von drei konkreten Beispielen für Geräte-Beschreibungen aus den einander konzeptionell sehr ähnlichen Lehrgedichten der neapolitanischen Mikrotradition des späteren siebzehnten und des früheren achtzehnten Jahrhunderts $^{21}$ veranschauliche, welche Möglichkeiten der Gestaltung und Verwendung dieses Formelements sich bieten.

Der erste Punkt betrifft den ,sachlichen Gehalt‘ beziehungsweise den ,Informationswert" der Beschreibungen. Ob die in einem Lehrgedicht enthaltenen Informationen sachrichtig und vollständig sind, ob sie den aktuellen Wissensstand zeigen oder ältere Diskurse reaktivieren, spielt für die Einschätzung eines Lehrgedichts

17 Giuseppe Maria Mazzolari: Electricorum libri VI. Rom 1767, S. 22-24.

18 Mazzolari, Electricorum libri, (Anm. 17) S. 65-67.

19 Mazzolari, Electricorum libri, (Anm. 17) S. 152-154.

20 Vgl. dazu das Programm der FOR 2305 „Diskursivierungen von Neuem“ unter http://www. for2305.fu-berlin.de/ (20. Februar 2020).

$21 \mathrm{Zu}$ dieser Mikrotradition vgl. Claudia Schindler: La cultura letteraria dei Gesuiti a Napoli (1680-1730): Tradizioni locali come propaganda per una rete mondiale. In: Per la valorizzazione del patrimonio culturale della Campania. Il contributo degli studi medio- e neo-Latini. Hg. von Giuseppe Germano. Neapel 2016 (Latinae humanitatis itinera nova 2), S. 117-127; dies.: Exploring the Distinctiveness of Neo-Latin Jesuit Didactic Poetry in Naples: The Case of Nicolò Partenio Giannettasio. In: Exploring Jesuit Distinctiveness. Interdisciplinary Perspectives on Ways of Proceeding within the Society of Jesus. Hg. von Robert A. Maryks. Leiden 2016 (Jesuit Studies 6), S. 24-40. 
grundsätzlich eine wichtige Rolle ${ }^{22}$ und ist auch bei der Betrachtung der Beschreibungen technischer Artefakte relevant. Dort kommt jedoch noch ein weiterer Aspekt hinzu. Zumindest äußerlich ist die neulateinische Jesuiten-Lehrdichtung des siebzehnten und achtzehnten Jahrhunderts eine konservative Gattung. ${ }^{23}$ Sie orientiert sich eng an den Modellen der klassischen römischen Lehrdichtung, insbesondere an Vergils Georgica, die in der neapolitanischen Mikrotradition wichtigster Referenztext sind. ${ }^{24}$ Das Vokabular, das den Lehrdichtern zur Verfügung steht, ist im Wesentlichen das poetische Vokabular der klassischen Latinität. Die sprachlichen Vorgaben sind somit a priori ungleich restriktiver als in den lateinischen Prosatraktaten oder gar in volkssprachlichen Abhandlungen. Die Frage nach dem sachlichen Gehalt bezieht sich daher nicht allein auf die Frage, inwieweit die Beschreibungen vollständig, sachrichtig und aktuell sind, sondern auch auf die Frage, ob und wie die Lehrdichter das Problem lösen, dass ihnen aufgrund von generischen Vorgaben eine Fachterminologie nicht oder nur sehr eingeschränkt zur Verfügung steht.

Mit der Entscheidung, die der Lehrdichter hinsichtlich des sachlichen Gehalts der Beschreibung trifft, ist eine weitere grundsätzliche Frage der antiken und neulateinischen Lehrgedichtpoetik verbunden. Es geht um die Frage, inwieweit die Vermittlung von Sach- und Faktenwissen alleiniges oder vorrangiges Ziel des Lehrgedichts ist, oder ob sich hinter den Sachinformationen weitere Dimensionen ausmachen lassen, die dem Lehrgedicht, um es in der Terminologie Bernd Effes zu formulieren, „Transparenz“ verleihen. ${ }^{25}$ Dass sich für die Lehrdichtungen der neapolitanischen Mikrokultur eine solche „Transparenz“ nachweisen lässt, steht außer Frage. Wie die übrigen Jesuiten-Lehrgedichte vermitteln auch die Lehrgedichte der Neapolitaner das Bild einer Welt, „informed, if not forged by the values of the society of Jesus“, wie es Yasmin Haskell formuliert hat. ${ }^{26}$ Intellektuelle Herausforderung und wissenschaftliche Studien, Arbeitsethos und Sorgfalt sind ebenso wichtige Ingredienzien des dort sich manifestierenden jesui-

22 Vgl. für das antike Lehrgedicht Bernd Effe: Dichtung und Lehre. Untersuchungen zur Typologie des antiken Lehrgedichts. München 1977 (Zetemata 69), der auf der Grundlage ,Verhältnis des Dichters zu seinem Stoff“ einen typologischen Ansatz erarbeitet.

23 Vgl. Yasmin A. Haskell: Loyola’s Bees: Ideology and Industry in Jesuit Latin Didactic Poetry. Oxford 2003, S. 14-16.

24 Vgl. Claudia Schindler: Wissen ist Macht! Nicolò Partenio Giannettasio (1648-1715) und die neulateinische Gelehrtenkultur der Jesuiten in Neapel. In: Scientia poetica 18 (2014), S. 28-59, hier S. 38-39.

25 Effe (Anm. 22), S. 40-79. Als Muster eines Lehrgedichts des ,transparenten‘ Typus gelten Effe die Phainomena des Arat, die auf der Oberfläche den Sternhimmel behandeln, dahinter aber das Wirken einer stoischen Allmacht sichtbar machen.

26 Haskell (Anm. 23), S. 13. 
tischen Weltverständnisses wie ein gewisser Anspruch auf Ordnung und Organisation. ${ }^{27}$ Da alle wissenschaftlichen Studien ad maiorem Dei gloriam geschehen, sind die Lehrgedichte stets mit einem christlichen Subtext unterlegt. Wenn der Mensch kreativ ist, wenn er verschiedene artes erfindet, nutzt er das geistige Potenzial, das ihm von Gott verliehen wurde. Die einzelnen Autoren stimmen überein, was diese Wertebasis angeht. Sie verhandeln sie jedoch in ihren Lehrgedichten nicht nur unter verschiedenen Oberthemen, sondern auch mit unterschiedlichen Akzentuierungen. Dabei erweisen sich gerade die Beschreibungen von Geräten und Maschinen als Kristallisationspunkte, die in ihrer spezifischen Machart wie auch der Präsentation und Kontextualisierung der Artefakte den Subtext des jeweiligen Lehrgedichts reflektieren können.

\section{Die Bilgepumpe}

Nicolò Partenio Giannettasios Nauticorum libri VIII, ein Lehrgedicht über Seefahrt und Schiffbau, ist das früheste der ,neapolitanischen' Lehrgedichte. 1686 in stark erweiterter zweiter Auflage in Neapel erschienen und 1715 letztmalig gedruckt, ${ }^{28}$ sind die Nautica ein enzyklopädisches Werk, das in acht Büchern und mehr als 8000 Hexametern sämtliche Aspekte der Seefahrt vom Bau eines Schiffes bis hin zu den transozeanischen Routen darstellt. ${ }^{29}$ Das sechste Buch behandelt in seinem ersten Teil das Verhalten im Sturm. In diesem Zusammenhang weist der Lehrdichter seinen Schüler auf die Notwendigkeit hin, das in den Kielraum eines leckgeschlagenen Schiffes eingedrungene Wasser zu entfernen. Im Anschluss daran stellt er die dafür erforderliche technische Vorrichtung vor (Nautica, 6. Buch, S. 171):

\footnotetext{
In primis facili torno, duraque cavandus

Ex ulmo tibi modiolus, pedibusque carinam

Ter quinis super ascendat: sic antlia fluctus

Evomere alta potest, aequorque remittere in aequor.

Hinc saeptum in medio transversum impone canali

Ex aere, aut ligno e solido: sed valvula circo

Insit aperta: cuproque illi mox insuper adde,
}

27 Haskell (Anm. 23), S. 6-13.

28 Giannettasio (Anm. 14), S. 49-250. Textgestaltung und Angabe der Seitenzahlen folgen dieser Ausgabe.

29 Vgl. Claudia Schindler: Nicolò Partenio Giannettasios Nauticorum libri VIII. Ein neulateinisches Gedicht des 17. Jahrhunderts. In: Neulateinisches Jahrbuch 3 (2001), S. 145-176, hier S. 149-150. 
aut durum e corio tectum, clavoque refige, concludi ut pariter possit, pariterque recludi, sive aditus sit dandus aquae, aut prohibendus ab illa est.

His actis, valido de robore necte canali

Embolum, et aere illum circum vestire necesse est.

Aeque et modiolo insertus respondeat imo:

Ne qua per latera intromissus permeet aer.

Est cavus ipse etiam formandus et ante cylindrus:

Cui saeptum, et medio sit valvula: et insuper olli

E corio tegumen sit mobile, quo fluat unda

Intromissa tubo, et clauso, non refluat inde.

Ferrea $^{30}$ firmandus manica mox embolus axi

Ferreus, et solidis insit versatilis axis

Cardinibus super, ut possit cavus embolus alto

Nunc facili attolli nisu, rursusque deorsum

Demitti, et potos sentina reddere fluctus.

At vero ut valeas axem versare rotundum,

Praelongam capulo virgam duro insere ferro.

Haec fuerit constructa tibi cum machina, ab ipsa

Momento poteris fluctus haurire carina:

Nam si demittas virgam, tollasque vicissim,

Continuo exhaustum ex imo dabit antlia pontum.

Zunächst musst du mit einem leicht drehenden Bohrer und aus hartem Ulmenholz einen Schaft aushöhlen, der 15 Fuß vom Kiel aus aufragt. So kann die Pumpe die Fluten in die Höhe ragend ausspeien, und Meerwasser ins Meer zurückbefördern. Dann installiere in der Mitte der Röhre ein Ventil ${ }^{31}$ aus Erz oder aus festem Holz: Aber die kreisrunde Öffnung soll eine Klappe haben, die sich öffnen kann. Füge jener bald darauf eine Abdeckung aus Kupfer oder aus hartem Leder hinzu, und befestige sie mit einem Zapfen, damit sie in gleicher Weise geöffnet wie geschlossen werden kann, sei es, dass die Wasserzufuhr gewährt oder verhindert werden muss. Wenn das geschehen ist, dann füge der Röhre einen Kolben aus Kernholz hinzu, und es ist nötig, ihn ringsum mit Erz zu umkleiden. In gleicher Weise soll ihm (ein zweiter Kolben) ganz unten in dem Schaft entsprechen, damit nicht seitlich irgendwo Luft durchdringen kann. Zuvor muss man auch noch einen hohlen Zylinder formen. Er soll ein Ventil und in der Mitte eine Klappe haben. Und über ihr soll eine bewegliche Bedeckung aus Leder sein, durch die das Wasser zwar in die Röhre einströmen kann, aber nicht mehr herausströmen, wenn sie geschlossen ist. Mit einem eisernen Haken muss der eiserne Kolben dann an einer Achse befestigt werden, und die drehbare Achse soll oben in festen Angeln stecken, damit der hohle Kolben mit wenig Anstrengung bald in die Höhe gehoben, bald gesenkt werden kann, und die aus der Bilge gesogenen Fluten abgeben kann. Aber damit man die runde Achse drehen kann, füge einen langen Schwengel aus hartem Eisen mit einem Handgriff an. Nachdem du diese Vorrichtung konstruiert hast, wirst du sogleich die Fluten geradewegs aus dem

30 ferrea (Abl. Sg. Fem.) muss mit Synizese gelesen werden.

31 saeptum transversum: eigentlich „Zwerchfell“ (Celsus 4,1,4). 
Kielraum saugen können: Denn wenn du den Schwengel im Wechsel senkst und hebst, dann wird die Pumpe fortwährend das ganz unten aus dem (Kielraum) gesaugte Meerwasser abgeben. ${ }^{32}$

Bei der Apparatur, die der Dichter beschreibt, handelt es sich um eine sogenannte Lenz- oder Bilgepumpe (antlia), eine im Kielraum des Schiffes fest installierte hydraulische Vorrichtung, die es ermöglicht, das Wasser aus dem Kielraum nach oben zu saugen. Die Beschreibung ist mit 29 Hexametern relativ umfangreich. Ihr Aufbau ist didaktisch geschickt: Giannettasio gestaltet sie als eine Art Bauanleitung, die das Objekt Schritt für Schritt in seiner Entstehung zeigt und abschließend nochmals in seiner Funktion vorführt. Gerundivkonstruktionen (cavandus), jussive Konjunktive (ascendat), Imperative (impone) oder Konstruktionen mit necesse est prägen die Darstellung. Die Syntax ist überwiegend parataktisch, die Bauschritte sind durch adverbiale Bestimmungen oder Einleitungsformeln markiert, die eine zeitliche Sukzession anzeigen: in primis, hinc, his actis, mox. Die einzelnen Teile der Pumpe werden in sinnvoller Reihenfolge präsentiert: Giannettasio schildert zunächst die Anfertigung eines Hohlrohrs (modiolus) als Pumpenschaft, sodann das untere Ventil (saeptum), den Kolben (embolus), den Zylinder (cylindrus) und das obere Ventil (tegmen), schließlich den Schwengel (virga) und den Handgriff (capulus), mit dem der Kolben auf und ab bewegt werden kann. Terminologisch bewegt sich die Beschreibung nahezu ausschließlich im Bereich der klassischen Latinität. Sämtliche Begriffe, die Giannettasio verwendet, sind in der Fachprosa belegt. Die Bezeichnung der Pumpe als antlia ist bereits antik. Ein Epigramm der Anthologia Latina bietet eine lebendige Beschreibung ihrer Funktion. ${ }^{33}$ Auch modiolus in der Bedeutung „Hohlrohr“, „Schaft“ ist bereits für die Antike nachweisbar. ${ }^{34}$ Eventuelle terminologische Unklarheiten fängt Giannettasio zum einen dadurch auf, dass er die Herstellung des Bauteils beschreibt. Der modiolus entsteht durch das Aushöhlen eines Ulmenholzes mit einem Bohrer; die anschließende Angabe, dass er vom Kiel aus fünfzehn Fuß aufragt, zeigt, dass es sich um ein längliches Objekt handeln muss, das senkrecht in das Schiff eingebaut wird. Die Darstellung wird zum anderen dadurch verständlich, dass auf die Nennung der einzelnen Teile jeweils ein Finalsatz folgt, der diese in ihrer Funktion erläutert: Der modiolus der Pumpe muss vom Kiel aufragen, damit er das geschöpfte Wasser überhaupt erst wieder abgeben kann. Das untere Ventil muss einen Verschluss haben, damit das Wasser nur bei Bedarf einströmt (concludi ut

32 Sämtliche Übersetzungen, soweit nicht anders angegeben, stammen von der Verfasserin.

33 Anth. Lat. 284: „fundit et haurit aquas, pendentes evomit undas, et fluvium vomitura bibit. mirabile factum. portat aquas, portatur aquis. sic unda per undas volvitur et veteres haurit nova machina lymphas.“

34 Vgl. TLL, vol. VIII, S. 1239 s.v. modiolus. 
pariter possit, pariterque recludi). Aus demselben Grund muss das obere Ventil mit einer Klappe ausgestattet sein; der Kolben (embolus) muss sich passgenau in den Pumpenschaft einfügen, ne qua per latera intromissus permeet aer. In ihrer sprachlichen Gestaltung wirkt Giannettasios Beschreibung insgesamt nüchtern und sachbezogen, beinahe prosaisch. Die qualifizierenden Adjektive dienen nicht dem ornatus. Sie betreffen ausschließlich die für die Einzelteile der Pumpe verwendeten Materialien, die für die Gesamtkonstruktion von entscheidender Bedeutung sind: Der Schaft ist aus Ulmenholz, das untere saeptum aus Erz oder aus festem Holz; das obere Ventil aus Leder. Für die Bezeichnung des Wassers verwendet Giannettasio vier Synonyme, die ebenfalls in der Prosa gebräuchlich sind: fluctus, aequor, unda, aqua; allein das letzte Wort der Beschreibung, pontus, ist (auch in seiner Stellung am Ende des Hexameters) poetisch. ${ }^{35}$

Insgesamt handelt es sich bei Giannettasios Beschreibung der Bilgepumpe um eine klar aufgebaute, in jedem Punkt sachbezogene Darstellung, die das Objekt als solches auch für Leser mit einem geringeren technischen Sachverstand identifizierbar macht. In auffälligem Kontrast zu dieser nüchternen Beschreibung stehen die Verse, in denen der Dichter das Thema des Abschnittes angekündigt hatte (Nautica, 6. Buch, S. 170):

\footnotetext{
Nec minor ille labor fluctus haurire refusos

Sentina ex humili, rimas laxare patentes

Plurima cum coepit pelago concussa carina.

Sed quibus epotos valeas educere fluctus

Artibus, expediam, compertaque mira docebo.
}

Und nicht geringere Anstrengung besteht darin, die einströmenden Fluten aus der niedrig gelegenen Bilge zu schöpfen, wenn der Schiffskörper, vom Meer erschüttert, begonnen hat, zahlreiche klaffende Lecks zu öffnen. Durch welche Künste aber du imstande sein wirst, die eingedrungenen Fluten hinaus zu befördern, das will ich darlegen, und dich über wundersame Erfindungen belehren.

Gegenüber dem Lehrabschnitt hat die Themenangabe eine deutlich elaboriertere Diktion. Diese zeigt sich sowohl an den komplexeren hypotaktischen Satzstrukturen als auch an der Verwendung des poetischen Wortes pelagus, an der Synekdoche carina für navis, an der Enallage plurima [...] carina und schließlich an der Verwendung von pleonastischen Epitheta (sentina ex humili; rimas patentes). Dass die beiden letzten Verse, sed quibus epotos valeas educere fluctus / artibus,

35 Vgl. TLL, vol. X 1, S. 2686-2690 s.v. pontus; 2686: „legitur in poesi inde ab Enn., Acc., saepe in fine versus; in orat (ione) soluta saepius in iunctura (pont)us Euxinus.“ 
expediam, mit der Verbform expediam wie auch mit dem daran angeschlossenen indirekten Fragesatz auf die lucrezische und vergilische Lehrdichtung verweisen, ${ }^{36}$ verstärkt die poetische Färbung. Inhaltlich zerfällt die Einleitung in zwei Teile: Während der erste Teil auf das leckgeschlagene Schiff und die nicht geringe Anstrengung fokussiert ist, das eingedrungene Wasser zu entfernen, kündigt der zweite Teil an, eine Lösung des Problems zu präsentieren. Dabei stehen sich als Kernbegriffe des Abschnittes labor auf der einen und artes beziehungsweise comperta mira auf der anderen Seite gegenüber. Alle drei Begriffe stehen für zentrale Aspekte jesuitischen Selbst- und Weltverständnisses: Labor für mit Anstrengung verbundene Tätigkeiten, die die Mitglieder des Ordens zu leisten, aber auch für die Herausforderungen, denen sie sich zu stellen haben. Artes und mira comperta hingegen sind, als Manifestationen intellektueller Tätigkeit, die jesuitische Antwort auf diese Herausforderungen. ${ }^{37}$ Es handelt sich um Leitbegriffe, die in den Nautica immer wieder wiederholt werden.

Der schlichte Tonfall der Beschreibung spiegelt die funktionale Ästhetik des Geräts wieder. Dass der Erfindung nach Ansicht des Dichters etwas Staunenswertes, beinahe schon Mirakulöses anhaftet, mag als Hinweis darauf zu lesen sein, dass der menschliche Intellekt zu diesen Leistungen nur deswegen fähig ist, weil er dem Menschen von Gott verliehen wurde. Letztlich manifestiert sich also in der Fähigkeit des Menschen, eine komplexe Apparatur wie die Bilgepumpe zu konstruieren, göttliche Vorsehung. Und auch dem Dichter kommt in diesem Zusammenhang eine wichtige Aufgabe zu: Seine exakte Darstellung dient vordergründig gewiss dazu, den Gegenstand zu beschreiben und in seiner Funktion zu erläutern. Letztlich erweist auch sie in ihrer Schnörkellosigkeit und ,Fachlichkeit` dem von Gott verliehenen menschlichen Erfindungsgeist ihre Reverenz. Sie vermag es, den Gegenstand so gekonnt in das Medium der Literatur umzusetzen, dass er wieder visualisierbar wird.

36 Z. B. Lucr. 2, 58-62; 4, 927-929; 6, 493-495; Verg. Georg. 4,148-149.

37 Haskell (Anm. 23), S. 70 spricht für Giannettasios Halieutica von „poetry of wonder“, zu den Nautica vgl. Schindler: Wissen ist Macht! (Anm. 24), S. 42. 


\section{Die Zuckerrohrmühle}

Intellekt und menschlicher Erfindungsgeist spielen auch in Tommaso Strozzis 1689 gedrucktem Lehrgedicht De mentis potu seu cocolatis opificio eine wichtige Rolle. ${ }^{38}$ Das ungewöhnliche, in seinem Verhältnis zu den übrigen Lehrgedichten der neapolitanischen Mikrokultur schwer bestimmbare ${ }^{39}$ und bibliographisch kaum nachweisbare Werk ${ }^{40}$ behandelt in seinen drei Büchern Herkunft, Verarbeitung und therapeutischen Nutzen von Schokolade, deren Konsum sich an den europäischen Adelshöfen im ausgehenden siebzehnten Jahrhundert wachsender Beliebtheit erfreute. Zur Veredlung des an sich bitteren Kakaos bedarf es des Zuckers, dessen Gewinnung aus Zuckerrohr der Dichter am Schluss des ersten Buches beschreibt (1,26-29). Es handelt sich dabei um ein komplexes, mehrschrittiges Verfahren, bei dem das Zuckerrohr zunächst zerkleinert und zerdrückt, dann ausgepresst, der gewonnene Saft mehrfach durch Erhitzen geläutert und schließlich in eine kristalline Form gebracht wird, die in konische Gefäße gefüllt und zu Zuckerhüten geformt wird. Dass technisches Gerät an der Zuckerproduktion einen wesentlichen Anteil hat, wird bereits in den einleitenden Versen deutlich (De cocolatis opificio, 1. Buch, S. 26):

Hoc (sc. sacchar), age, quae calamis, operoso machina nisu,

Exprimat, et notae solertia construat artis,

Accipe:

Vernimm nun, welche Maschine mit arbeitsreicher Anstrengung den Zucker aus den Halmen presst und welche Geschicklichkeit bekannter Kunstfertigkeit ihn auftürmt.

Ähnlich wie Giannettasio nutzt Strozzi die Themenangabe, um den Duktus der nachfolgenden Beschreibung zu akzentuieren. Zentral ist auch bei ihm die Wahrnehmung der Maschine als Artefakt und des bearbeiteten Zuckers als Produkt menschlicher Geschicklichkeit, notae solertia artis. Gegenüber Giannettasio kommen jedoch zwei weitere Aspekte hinzu. Die machina erhält den Zusatz operoso nisu. Operoso, „voll von Betätigung“, verweist dabei auf den aufwändigen und lang-

$38 \mathrm{Zu}$ dem Gedicht ausführlicher Haskell (Anm. 23), S. 82-101; dies.: Poetry or Pathology? Jesuit Hypochondria in Early Modern Naples. In: Early Science and Medicine 12 (2007), S. 187213, hier S. 192; dies.: Bad Taste in Baroque Latin? Father Strozzi’s poem on Chocolate. In: Tous vos gens à latin: le latin, langue savante, langue mondaine (XIVe-XVIIe siècle). Hg. von Emmanuel Bury. Genf 2005, S. 429-438; Claudia Schindler: Der Kakao: Ein europäisches Getränk? Luxus, Rausch und Wirksamkeit in Tommaso Strozzis De mentis potu sive de cocolatis opificio (1689) (im Druck).

39 Vgl. Haskell: Poetry or Pathology? (Anm. 38), S. 192.

40 Vgl. Schindler: Der Kakao (Anm. 38) (im Druck). 
wierigen Arbeitsprozess, der Fleiß und Betriebsamkeit erfordert. Mit nisus, „Anstrengung“, wird ein weiterer Punkt berührt: Das gesamte Verfahren ist nicht nur intellektuell fordernd und arbeitsaufwändig, sondern erfordert auch physische Kraft.

In der anschließenden Beschreibung der Zuckergewinnung finden sich alle drei Punkte wieder, die die Einleitung ouvertürenartig antizipiert hatte. Die Ankündigung des Dichters, über eine machina informieren $\mathrm{zu}$ wollen, ,die den $\mathrm{Zu}$ cker aus den Halmen presst“ (quae hoc (sc. sacchar) e calamis exprimat) ist etwas irreführend, denn es ist nicht die Presse, sondern die Zuckerrohrmühle, die der Dichter im Folgenden in einer ausführlicheren Beschreibung präsentiert. Die übrigen für die Produktion erforderlichen Gerätschaften (Presse, Siedekessel und Zuckerhutform) werden genannt und in ihrer Funktion im Herstellungsprozess gezeigt, aber nicht näher beschrieben. Die Zuckerrohrmühle nun ist Teil des zweiten Arbeitsschrittes, des Zerkleinerns des Zuckerrohrs und der Vorbereitung für das Auspressen. Dass ihre Beschreibung gegenüber der Beschreibung der Bilgepumpe bei Giannettasio deutlich reduziert ist, zeigt bereits die Tatsache, dass sie nur 11 Verse von den etwa 70 Versen einnimmt, die den gesamten Prozess der Zuckerproduktion schildern (De cocolatis opificio, 1. Buch, S. 26-27):

Pars operi sectos aptant, saxoque molari

Vndique substernunt impacta mole terendos.

Saxea descripto sese explicat area gyro,

Cui mediae laxo stipes versatilis orbe:

Figitur, elato sursum qui vertice prodit.

Nectitur huic, vinclis adstricta trabalibus, ingens

Orbita, dissecto scopuli caesa orbita dorso;

Quae verso circum, multa vi, stipite, raptim

Ipsa etiam multos ocyssima circinat orbes.

Hinc molles subigit calamos, tenuatque subactos,

Comminuitque, molitque; inito nec fervida cursu

Absistit; donec cumulum se mutua in unum

Constipent, presso coalentia frustula visco.

Ein Teil (der Arbeiter) bereitet die zerschnittenen Halme für die weitere Verarbeitung vor, und von allen Seiten schieben sie sie unter den Mühlstein, damit sie durch die aufgebürdete Masse zerrieben werden können. Eine steinerne Fläche, kreisrund, tut sich auf. In ihrer Mitte wird eine in geräumigem Rund drehbare Stange festgesetzt, die mit erhabener Spitze in die Höhe ragt. Mit ihr durch balkendicke Klammern fest verbunden wird ein riesiger Mühlstein: ein Mühlstein, der aus der herausgeschnittenen Wölbung eines Felsvorsprungs herausgehauen wurde. Wenn die Stange mit viel Kraftaufwand in eilige Bewegung versetzt wird, dann dreht dieser selbst sehr schnell zahlreiche Kreise. Dadurch zerarbeitet er die Halme, dass sie weich werden, und macht sie platt, nachdem sie ihm untergeschoben wurden; macht sie dann noch dünner, und zermahlt sie. Und glühend heiß lässt er nicht ab vom einmal begonnenen Lauf, solange bis sich die Brocken zu einem Klumpen ballen, wenn sie der herausgepresste Sirup zusammenklebt. 
Strozzis methodisches Vorgehen in der Beschreibung ähnelt dem von Giannettasio: Er beschreibt zunächst die einzelnen Teile der Mühle (eine kreisrunde Fläche mit einer drehbaren Stange in der Mitte, auf die der Mühlstein aufgesetzt und mit einer Balkenkonstruktion verbunden wird), um im Anschluss daran das Gerät in Funktion zu zeigen. Gleichwohl unterscheidet sich seine Ekphrasis-Technik nicht unerheblich von der seines Vorgängers. Anders als Giannettasios Beschreibung sind Strozzis Ausführungen nicht adhortativ, sondern deskriptiv, wie überhaupt der gesamte Produktionsvorgang nicht in einem appellativen Lehrvortrag präsentiert wird, sondern in einer Narration, die durch eine temporale Sukzession geprägt ist und bei der die als vernae bezeichneten Plantagen-Arbeiter Protagonisten der Handlung sind. Insgesamt vermittelt zwar auch Strozzis Darstellung einen guten Eindruck von der Konstruktion. Doch ist die vorgestellte machina technisch wenig anspruchsvoll. Sie besteht lediglich aus drei Teilen: Grundfläche (area), drehbare Stange (stipes versatilis) und Mühlstein (orbita). Strozzi beschreibt zudem nicht oder zumindest nicht erkennbar die Ende des siebzehnten Jahrhunderts in der Zuckerproduktion eingesetzte Drei-Walzen-Mühle, wie sie auf zeitgenössischen Abbildungen gezeigt wird, ${ }^{41}$ sondern ein unspezifisches Mahlwerk, wie es bereits in der Antike bekannt gewesen sein könnte: Die machina wird also nicht als ,neu', sondern als ,alt‘ markiert. Gegenüber Giannettasios nüchterner Schilderung weist Strozzis Darstellung eine deutliche poetische Färbung auf. Der Satzbau ist überwiegend hypotaktisch mit mehreren Graden der Unterordnung. An mehreren Stellen finden sich Enjambements (orbe / figitur; ingens / orbita; cursu / absistit), die die kontinuierliche Bewegung des Mahlwerks nachzuzeichnen scheinen. Eher denn auf die Komplexität der Maschine kommt es dem Dichter auf ihre Größe und ihre Wuchtigkeit an, die in der steinernen Grundfläche (saxea area), den „balkenstarken Klammern“ (vincla trabalia), dem riesigen Mühlrad (ingens / orbita), das aus einem Felsvorsprung herausgeschnitten wurde (dissecto scopuli caesa orbita dorso), zum Ausdruck kommt. Das Augenmerk des Dichters gilt weiterhin der Schnelligkeit der Mechanik (raptim; ocissima) und der Intensität und Kontinuität ihrer Bewegung (inito nec fervida cursu / absistit), schließlich der Kraftanstrengung, die erforderlich ist, um den Mühlstein in Bewegung zu setzen (verso [...] multa vi stipite). Akustische Eindrücke fehlen hingegen vollständig. Das ist auffällig, denn eine Apparatur wie diese erzeugte wahrscheinlich ohrenbetäubenden Lärm. Die Beschreibung zielt offensichtlich nicht auf eine realistische Abbildung. Sie ästhetisiert die durch Sklavenarbeit betriebene, laute und schmutzige Arbeit der Zuckergewinnung und stilisiert sie zu einer harmonischen Kombination

41 Das Deutsche Technikmuseum besitzt ein Exemplar einer solchen Drei-Walzen-Mühle aus Bolivien (um 1700). 
von Erfindergeist, Arbeitseifer und Anstrengung. Das Schneiden und Zerkleinern des Zuckerrohrs wird zu einer minutiös geplanten Choreographie, zu einem exemplum für das Zusammenwirken von Mensch und Maschine, das durch die Anwendung jesuitischer Kardinaltugenden ermöglicht wird.

\title{
3 Das Wasserhebewerk
}

Camillo Eucherio De Quinzis Inarime, 1726 gedruckt, ist das späteste Lehrgedicht der neapolitanischen Mikrotradition. Gegenstand der sechs Bücher sind die Heilquellen der Insel Ischia, ihre Badekultur und der medizinische Nutzen ihrer Anwendungen. ${ }^{42}$ Das zweite Buch bietet eine Übersicht über Bäder in Italien, Europa und dem Mittelmeerraum sowie einen historischen Abriss über die Entwicklung der Badekultur in der Antike, die ihren Höhepunkt in den römischen Thermenanlagen findet. Dabei kommt der Dichter auch auf die technischen Aspekte der Thermen zu sprechen. Nach der Beschreibung der Hypokaustenanlage kündigt er an, den Mechanismus beschreiben $\mathrm{zu}$ wollen, der es ermöglicht, das Wasser mit hohem Druck nach oben zu transportieren (86-88). Es handelt sich dabei um eine antike Erfindung, wie sie Vitruv in ähnlicher Form als Wasserdruckwerk des Ktesibios im zehnten Buch von De architectura beschrieben hatte. ${ }^{43}$ Sie wird vom Dichter gleichwohl als ,neu“ diskursiviert: (Inarime, 2. Buch, 86-87):

\footnotetext{
At quo tracta modo supera in convexa domorum

Repserit: elatas et lympha insperserit aedes

Nosse velis si forte: meo te carmine Paean

Instruet, et doctum mox per non cognita ducet.
}

\begin{abstract}
Aber wenn du vielleicht wissen möchtest, auf welche Weise das Wasser hochgezogen wurde und in die oberen Wölbungen der Baulichkeiten strömte und das hochragende Gebäude von oben herab benetzte, dann wird Apollo dich durch mein Gedicht informieren, und dich bald durch Unbekanntes führen und belehren.
\end{abstract}

42 Camilli Eucherii de Quintiis: Inarime seu de balneis Pithecusarum libri VI. Neapel 1726. Eine italienische Übersetzung des Textes mit ausführlicher Einleitung bietet: Camillo Eucherio de Quintiis: Inarime (De balneis Pithecusarum), traduzione di R. Castagna. Ischia 2003. Zu De Quinzi außerdem Gennaro Gamboni S.J.: Ischia e il suo poeta, Camillo Eucherio Quinzi S.J. Neapel 1952; Antonietta Iacono: Gli umanisti e le acque di Ischia. In: Intorno ai Campi Flegrei: Memorie dell'acqua e della terra. Hg. von Rossana Valenti. Neapel 2011, S. 63-77, hier S. 74-75.

43 Vitr. 10,7. 
Der Dichter kündigt seinem Leser an, ihn in seinem Gedicht durch Unbekanntes (per non cognita) führen und so belehren zu wollen. Die anschließende, gut 30 Verse umfassende Darstellung beschreibt das Wasserdruckwerk in seinen wesentlichen Punkten (Inarime, 2. Buch, S. 86-88) ${ }^{44}$ :

Namque nova ut tandem moles educta sub auras

Exierat, labor is primum impendendus, ut imis

Eruta vena cavis non una aspergine sursum

Tenderet, et summi peteret fastigia tecti.

Quippe suis postquam scatebris emissa propinquos

Fluxerat in nilos, et laxa liber habena

Pervia paulatim Castella ${ }^{45}$ expleverat humor;

Omnia tum tractos partiri cura liquores

Per loca: difficiles, et quas natura negavit

Solicita decet arte vias, aditusque sagaci

Ingenio stravisse: rotis iam machina stridet

Versa suis: radiisque gemunt agitata volutis

Tympana: iamque avido plenas siphone lacunas

Haurit ab extremo, dum traditur, antlia fundo.

Sic tubulis excepta, tenet iam pensilis altum

Paene oblita sui: iam scandit, et aera vincit

Unda sequax ducto qua provocat Orbita gyro.

Tunc etenim adversis violentia motibus actam

In sublime rapit: tectique ascendere culmen

Cogit: et invitam tollens hac ducit, et illac.

At quia nam tanto levior pervadat in arces

Impete, et aeriae feriat laquearia molis;

Seu quod pressa novo gravioris pondere caeli

Lympha solo assiliat: spatio seu territa inani

Nubiferos ierit conata irrumpere fines:

Dicite vos, rauci oblectant quos irrita Circi

Iurgia, clamoso passim exaudita theatro.

Quo se cumque modo nostrae sententia menti

Certa probet; superas nativi ponderis expers

Itque, reditque vias, et amico gurgite Naias

Interclusa cadit, plenoque interfluit imbri,

In quascumque velis diverso e tramite partes.

Hinc etenim ceu fonte suas Miliaria lymphas

Accipiunt, lapsosque bibunt ut ab aethere rores.

Denn sobald schließlich eine neue Wassermasse ans Licht gekommen war, musste man sich zuerst darum bemühen, dass die aus tiefsten Hohlräumen emporgeführte

44 Die im Original beigegebenen Fußnoten werden nicht mit abgedruckt.

45 Die Großschreibung bestimmter Begriffe entspricht dem Original. 
Quellader nicht in einem einzigen Strahl nach oben strebte und den obersten Punkt des Daches zu erreichen versuchte. Ja, nachdem das Wasser, aus seinen Quellen entlassen, in die nächsten Kanäle geflossen war, und das Nass frei, mit lockerem Zügel die Durchgangs-Reservoirs allmählich gefüllt hatte, da galt die Sorge, die hochgesogenen Wassermassen auf alle Orte zu verteilen: Es ist angemessen, schwierige Wege und Zugänge, die die Natur verweigert hat, mit unermüdlicher Kunstfertigkeit und mit scharfsinnigem Verstand zu eröffnen: Schon knirscht die Maschine, die sich mit ihren Rädern dreht. Von ihren sich drehenden Speichen getrieben, ächzen die Heberäder. Und während das Wasser weitergeleitet wird, schlürft schon die Pumpe mit gierigem Steigrohr die vollen Hohlräume aus unterster Tiefe aus. So, von den Röhren aufgenommen, hält es schon schwebend die Höhe, beinahe seiner selbst vergessend. Schon steigt die Woge auf und bezwingt die Luft. Sie folgt, wohin sie das $\operatorname{Rad}^{46}$ mit ihrer Umdrehung lenkt. Dann reißt sie die ungestüme Kraft mit gegenläufigen Bewegungen in die Höhe, und zwingt sie, den Dachfirst zu erklimmen. Und gegen ihren Willen hebt sie sie hoch und führt sie hierhin und dorthin. Aber, weshalb sie aufgrund von einem so starken Schwung leichter oben ins Gebäude gelangt und die Decke von luftiger Masse trifft: Sei es, weil das Nass nun, von dem neuen Gewicht schwererer Luft bedrängt, vom Boden abprallt oder es, durch ein Vakuum erschreckt, dahinströmte und versuchte, in die wolkentragenden Gefilde einzubrechen - das kündet, ihr, die die nutzlosen Zänkereien im rautönenden Hörsaal erfreuen, die man im lärmreichen Theaterrund weithin hörte. Auf welche Weise auch immer eine sichere Interpretation sich unserem Verstand bestätigen mag: Ohne sein angestammtes Gewicht fließt das Wasser auf den oberen Bahnen hin und her. Mit freundlichem Strudel fällt die Naiade dann hinab, wenn man ihr den Weg versperrt, und mit vollem Guss fließt sie von unterschiedlichen Wegen her in alle Richtungen, die du möchtest. Von hier empfangen die Warmwasser-Aufbereiter ihr Nass wie aus einer Quelle, und trinken es wie Tau, der vom Himmel herabgefallen ist.

Der Schwerpunkt der Darstellung liegt diesmal nicht auf der Apparatur an sich, sondern auf ihrer Funktion. Die Beschreibung ist wiederum linear: Nachdem das Wasser aus den Aquaedukten in die Reservoirs verteilt worden ist, wird es durch Schöpfräder, Steigrohre und Pumpen nach oben befördert und von dort aus in die Warmwasseranlagen der Thermen weiterverteilt. Der Abschnitt enthält aus Vitruv und Seneca bekannte Fachbegriffe des römischen Wasserwesens, wie castella (Reservoirs) ${ }^{47}$ und miliaria (Gefäße zur Warmwas-

46 Orbita soll hier synonym mit rota sein, wie der Dichter selbst S. 87 in einer Fußnote anmerkt.

47 Vitr. 8,6,1: „cumque venerit aqua rivis ducta ad moenia, efficiatur castellum et castello coniunctum ad recipiendam aquam triplex immissarium, collocenturque in castello tres fistulae; 8,6,4 sin autem fistulis plumbeis ducetur, primum castellum ad caput struatur, deinde ad copiam aquae lumen fistularum constituatur eaeque fistulae e castello collocentur ad castellum quod erit in moenibus.“ Ein gut erhaltenes castellum wurde in Pompeji ergraben, vgl. Christoph 
serbereitung), ${ }^{48}$ sowie des Ingenieurswesens, wie tympana (Heberad), sipho (Steigrohr), antlia (Pumpe). Der Dichter strebt in der Beschreibung Verständlichkeit an. Zentrale Begriffe erläutert er in Fußnoten, um sie dem nicht technikaffinen Leser zugänglich zu machen: So erklärt er die tympana als machina ad rotae similitudinem, qua a balneatoribus [...] aquae in superna vasa [...] infundebantur. Die Identifikation der als machina bezeichneten Konstruktion als La tromba dient dabei nicht nur dem Verständnis, sondern nähert die antike Konstruktion zeitgenössischer Technik an. Insgesamt jedoch treten in De Quinzis Beschreibung technische Aspekte in den Hintergrund. Der Dichter gesteht sogar freimütig ein, dass ihn die physikalischen Zusammenhänge, die das Aufsteigen des Wassers ermöglichen, nicht interessieren. Ob nun physische Kraft und Schwung, der Luftdruck oder ein Unterdruck für das Aufsteigen des Wassers verantwortlich sei, das sollten die erörtern, „die die nutzlosen Zänkereien im Hörsaal“ erfreuten (oblectant quos irrita circi / iurgia) - offensichtlich ein Seitenhieb auf öffentlich und nur um der Schau willen geführte wissenschaftliche Debatten. Im Vordergrund steht vielmehr ein emotionaler Aspekt. Wie seine Vorgänger betont auch De Quinzi die intellektuelle Herausforderung, die mit der Erfindung verbunden sei. Das aus Plinius bekannte ${ }^{49}$ sagax ingenium erlaube es, schwierige Wege zu öffnen, die die Natur verweigert habe: difficiles, et quas natura negavit / sollicita decet arte vias, aditusque sagaci / ingenio stravisse. Der gesamte Abschnitt ist zwar durch gliedernde Partikeln (nam, iam, iamque, at und hinc) wie ein wissenschaftlicher Vortrag strukturiert, erhält aber durch die lexikalische Gestaltung (etwa in den Bezeichnungen des Wassers als humor, liquores, unda oder lympha) eine poetische Färbung. Insgesamt ist die Darstellung als Auseinandersetzung zwischen Technik und Natur inszeniert, bei der die Technik die Natur $\mathrm{zu}$ ihrem eigenen Besten unter Kontrolle bringt und gefügig macht. Akustische Eindrücke, wie das Knirschen der machina und Ächzen der Heberäder (stridet, gemunt) charakterisieren die Anstrengung, die mit dem Prozess verbunden ist. Auffällig ist weiterhin eine Anthropomorphisierung der Kontrahenten ,Wasser ${ }^{\star}$ und ,Maschine‘. Die Pumpe erscheint als eigenständig handelndes, durstiges Lebewesen, das das Wasser gierig trinkt (haurit), es mit der Gewalt seiner Bewegungen in

P.J. Ohlig: Vitruvs 'castellum aquae' und die Wasserversorgung im antiken Pompeji. In: Schriftenreihe der Frontinus-Gesellschaft 19 (1995), S. 124-147.

48 Sen. Nat. 3,24,2: „dracones et miliaria et conplures formas, in quibus aere tenui fistulas struimus per declive circumdatas, ut saepe eundem ignem ambiens aqua per tantum fluat spatii, quantum efficiendo calori sat est“; 4,9: „minora balnearia et minora miliaria citius calefiunt“. Weitere Belege TLL vol. VIII, S. 948. Zu den römischen miliaria allgemein vgl. Joachim Marquardt: Das Privatleben der Römer. Leipzig 1886, S. 288.

49 Plin. Nat. 26,12; 33,17. 
die Höhe reißt (rapit) und es nach oben zwingt (cogit). Auf der anderen Seite tritt das Wasser als handelndes Subjekt auf, das „folgsam aufsteigt und die Luft besiegt“: scandit et aera vincit / unda sequax. Die Darstellung weist dem Wasser sogar etwas wie ein Bewusstsein und einen eigenen Willen zu; es kann ,sich beinahe selbst vergessen“ (pene oblita sui) oder „unwillig sein“ (invitam). Schließlich wird der Pumpvorgang als eine Art Befreiung inszeniert, bei der das Wasser das „ihm angeborene Gewicht“ verliert (nativi ponderis expers) und sich sogar in eine freundliche Naiade verwandelt (Naias amico gurgite), die sich nach Belieben überall hin verteilen lässt. Der Mensch tüftelt das Wasserdruckwerk also zwar kraft seines sagax ingenium aus. In den anschließend beschriebenen Abläufen spielt er jedoch keine Rolle mehr, so dass sich die Maschine von ihrem Erfinder gleichsam emanzipiert und autonom zu handeln scheint. Eine derartige Autarkie hatten weder Giannettasio noch Strozzi den von ihnen beschriebenen Konstruktionen zugesprochen. Steht also hinter De Quinzis Darstellung möglicherweise sogar ein generelles Plädoyer für die Autonomie des Produkts der Schöpfung von ihrem Schöpfer, das den aufklärerischen Gedanken des achtzehnten Jahrhunderts in jesuitischem Gepräge entgegenkommen will? 
